\title{
mUC: mehr Lebensqualität durch PD-1-Inhibitor in der Zweitlinie
}

\author{
Die Therapieoptionen für \\ Patienten mit metastasiertem \\ Urothelkarzinom (mUC) bleiben \\ beschränkt. Versagt die initiale \\ platinbasierte Chemotherapie, \\ kann Pembrolizumab die \\ Überlebenszeit verlängern - bei \\ verbesserter Lebensqualität.
}

\begin{abstract}
atienten mit einem mUC erhalten in der Erstlinie standardmäßig eine Chemotherapie auf Platinbasis. Das mediane Gesamtüberleben (OS) liegt für Carboplatin-Kombinationen bei nur neun und für Cisplatin-Kombinationen bei zwölf bis 15 Monaten. Für das weitere Vorgehen nach Therapieversagen bestehen derzeit keine international akzeptierten Empfehlungen. Neben Paclitaxel und Docetaxel als Monotherapie ist in Europa Vinflunin zugelassen.

Monoklonale Antikörper gegen PD-1 und seine Liganden PD-L1 und PD-L2
\end{abstract}

zeigten eine robuste antitumorale Aktivität bei fortgeschrittenen Malignomen, unter anderem beim UC. Im Rahmen der Phase-III-Studie KEYNOTE-052 wurde der Effekt des PD-1-Antikörpers Pembrolizumab auf OS und progressionsfreies Überleben (PFS) mit dem Effekt einer Monotherapie mit einem Taxan oder Vinflunin nach Versagen der Erstlinienbehandlung verglichen.

542 Patienten mit histologisch oder zytologisch gesichertem UC und Progress innerhalb von 12 Monaten nach der initialen platinbasierten Polychemotherapie erhielten randomisiert Pembrolizumab (200 mg/kg KG) oder ein Zytostatikum nach Wahl des behandelnden Arztes. Für die gesamte Studienpopulation ermittelten die Forscher ein medianes OS von 10,3 Monaten unter Pembrolizumab und 7,4 Monaten in der Vergleichsgruppe $(\mathrm{p}=0,002)$. Beschränkt auf die Gruppe mit positiver PD-L1-Expression auf Tumor- und infiltrierenden
Immunzellen ( $\geq 10 \%$ ) lagen die Überlebenszeiten in der PembrolizumabGruppe bei acht Monaten und in der Chemotherapiegruppe bei 5,2 Monaten $(p=0,005)$. Es fanden sich keine signifikanten Unterschiede bezüglich des PFS in den Gruppen, unabhängig vom Status der PD-L1-Expression. Patienten der Pembrolizumab-Gruppe hatten deutlich weniger therapieassoziierte Nebenwirkungen und auch weniger Nebenwirkungen vom $\mathrm{Grad} \geq 3$.

Fazit: Pembrolizumab als Zweitlinientherapie bei platinrefraktären Patienten mit einem fortgeschrittenen Urothelkarzinom erhöht signifikant das Gesamtüberleben und ist besser verträglich als traditionelle Zytostatika.

Wolfgang Zimmermann

Bellmunt J et al. Pembrolizumab as second-line therapy for advanced urothelial carcinoma. $\mathrm{N}$ Engl J Med. 2017; 376(11): 1015-26.

\section{Kein längeres Überleben durch Ipilimumab beim frühen metastasierten Prostatakarzinom}

\begin{abstract}
Ipilimumab (Ipi) ist ein Immuncheckpointhemmer, der sich gegen CTLA-4 ("cytotoxic T lymphocyte-associated antigen 4") richtet. Nach positiven Hinweisen aus Phase-I/II-Studien wurde Ipi in einer Phase-III-Studie bei Patienten mit asymptomatischem oder minimal symptomatischem metastasiertem kastrationsresistentem Prostatakarzinom (MCRPC) mit Placebo verglichen.
\end{abstract}

W ährend die Effektivität von Ipi beim metastasierten Melanom durch Phase-III-Studien eindeutig belegt ist, war der Stellenwert beim mCRPC unklar. 602 Patienten mit Progress unter laufender antihormoneller Therapie und gutem Allgemeinzustand (ECOG-Performancestatus [PS] 0-1), einem Testosteronwert $<50 \mathrm{ng} / \mathrm{dl}$ und geringen bis mäßigen Schmerzen erhielten 2: 1 randomisiert Ipi oder Placebo. Stratifiziert wurde unter anderem nach Allgemeinzustand, $\mathrm{LDH}$ und Schmerzen. Die Patienten erhielten $10 \mathrm{mg} / \mathrm{kg}$ Ipi oder Placebo alle drei Wochen (bis zu viermal). Patienten ohne Progress bekamen als Erhaltungsthera- pie alle zwölf Wochen Ipi oder Placebo bis zum Progress oder dem Auftreten inakzeptabler Nebenwirkungen. Primärer Endpunkt war das Gesamtüberleben (OS), sekundäre Endpunkte unter anderem progressionsfreies Überleben (PFS), Zeit bis zur nächsten zytotoxischen Therapie oder Schmerzprogression.

Hinsichtlich des medianen OS unterschieden sich die Studienarme nicht (28,7 vs. 29,7 Monate unter Placebo), auch bei keiner der Subgruppen. Das PFS war aber unter Ipi signifikant länger (5,6 vs. 3,8 Monate; Hazard Ratio 0,67, 95,87\%-Konfidenzintervall 0,55-0,81). Ein PSA-Ansprechen wurde unter Ipi häufiger erzielt als unter Placebo (25 vs. $8 \%)$. Therapiebedingte Nebenwirkungen traten unter Ipi deutlich häufiger auf als unter Placebo (82 vs. $49 \%$ ), schwerere Nebenwirkungen (Grad 3/4) bei 40 bzw. $6 \%$ der Patienten. Führend war hier eine Diarrhö (15 vs. 0\%). Alle anderen Grad-3/4-Nebenwirkungen waren vergleichsweise selten ( $\leq 3 \%$ der Patienten). An therapiebedingten Nebenwirkungen starben neun Patienten (2\%) im Ipi-Arm. Immunologische Nebenwirkungen vom Grad 3/4 traten bei $31 \%$ der Patienten unter Ipi und bei $2 \%$ unter Placebo auf.

Fazit: Durch Ipi konnte das Überleben beim mCRPC ohne oder mit geringen Symptomen nicht verlängert werden. Es kam jedoch zu einem leichten, aber signifikanten Anstieg im PFS und zu einer höheren PSA-Remissionsrate als unter Placebo.

Brigitte Schalhorn

Beer TM et al. Randomized, double-blind, phase III trial of ipilimumab versus placebo in asymptomatic or minimally symptomatic patients with metastatic chemo-naïve castration-resistant prostate cancer. J Clin Oncol. 2017; 35(1): 40-7. 